\title{
Terapia ocupacional e acidente vascular cerebral: revisão integrativa da literatura ${ }^{1}$
}

\author{
Carolina Gina Shin, Rosé Colom Toldrá \\ Departamento de Fisioterapia, Fonoaudiologia e Terapia Ocupacional, Faculdade de Medicina, Universidade de
} São Paulo - USP, São Paulo, SP, Brasil.

\begin{abstract}
Resumo: O presente estudo objetivou a sistematização do conhecimento produzido pela Terapia Ocupacional relacionado ao tema acidente vascular cerebral, nos últimos dez anos (2003-2013). Trata-se de uma revisão integrativa nacional da literatura realizada a partir das bases de dados: Scientific Eletronic Library Online - SciELO Brazil e Biblioteca Virtual em Saúde e Literatura Latino-Americana e do Caribe de Ciências da Saúde - LILACS. Foram encontrados 17 artigos sobre o tema estudado. Identificou-se que há uma maior tendência nas produções nacionais voltadas para a categoria de Estratégias e Intervenções Terapêuticas do que a Fundamentação Teórica e a Análise da Prática. Os estudos demonstraram que a atuação do terapeuta ocupacional proporciona melhora no desempenho funcional, na independência e na qualidade de vida aos pacientes com AVC. A produção científica recente em Terapia Ocupacional sobre o tema AVC contribuiu para maior divulgação e melhor desenvolvimento de práticas terapêuticas voltadas ao cuidado às pessoas com AVC e para um maior reconhecimento da necessidade e da importância do terapeuta ocupacional pelos demais profissionais. Recomenda-se uma produção mais diversificada, para atender aos padrões científicos internacionalmente reconhecidos, indo ao encontro da tendência das práticas de saúde. O estudo aponta a necessidade de intensificar a realização de pesquisas para divulgação do conhecimento produzido pela Terapia Ocupacional e seu reconhecimento no campo da reabilitação de pessoas com AVC.
\end{abstract}

Palavras-chave: Terapia Ocupacional, Acidente Vascular Cerebral, Literatura de Revisão, Pessoas com Deficiência, Reabilitação.

\section{Occupational therapy and stroke: literature integrative review}

\begin{abstract}
The present study aimed the systematization of knowledge produced by occupational therapy involving stroke within the last ten years (2003-2013). This is a national integrative literature review performed using the database: Scientific Eletronic Library Online - SciELO Brazil, Biblioteca Virtual em Saúde and Literatura Latino-Americana e do Caribe de Ciências da Saúde - LILACS. There were 17 articles on the topic studied. We identified a greater tendency in national productions facing the category Strategies and Therapeutic Interventions than the Theoretical Foundation and Analysis of Practice. Studies have shown that the performance of the occupational therapist improves functional performance, independence and quality of life for stroke patients. The recent occupational therapy scientific literature in contributed to greater disclosure and better development of therapeutic practices aimed to treat people with stroke and for greater recognition of the need and importance of occupational therapist by other professionals. We recommend a more diversified production, to meet the internationally recognized scientific standards, meeting the trend of health practices. The study points out the need to strengthen the researches to spread the knowledge produced by occupational therapy and its recognition in the field of rehabilitation of persons with stroke.
\end{abstract}

Keywords: Occupational Therapy, Stroke, Review, Disabled People, Rehabilitation.

\footnotetext{
Autor para correspondência: Rosé Colom Toldrá, Curso de Terapia Ocupacional, Departamento de Fisioterapia, Fonoaudiologia e Terapia Ocupacional, Faculdade de Medicina, Universidade de São Paulo, Rua Cipotânea, 51, Cidade Universitária, Butantã, CEP 05360-000, São Paulo, SP, Brasil, e-mail: rosetoldra@usp.br
}

Recebido em Dez. 27, 2014; $1^{\text {a }}$ Revisão em Mar. 18, 2015; $2^{\mathrm{a}}$ Revisão em Mar. 26, 2015; Aceito em Set. 15, 2015. 


\section{Introdução}

A transformação do perfil demográfico e socioeconômico da população brasileira é um importante fenômeno, marcado durante os meados do século XX. Esse processo de mudança esteve intimamente ligado com o panorama epidemiológico, que repercutiu no novo quadro de predomínio de doenças crônicas. Dentre as doenças crônicas não transmissíveis, destacam-se as doenças cardiovasculares, em particular o acidente vascular cerebral (AVC) ${ }^{2}$, que se apresenta, segundo os dados do Instituto Brasileiro de Geografia e Estatística - IBGE (INSTITUTO..., 2009), como a primeira causa de morte e de incapacidade no Brasil, o que gera grande impacto econômico e social.

Conforme definição recomendada pela Organização Mundial de Saúde - OMS (ORGANIZAÇÃO..., 2005), o AVC é um comprometimento neurológico focal (ou, às vezes, global), de ocorrência súbita e duração de mais de 24 horas - ou que causa morte - e de provável origem vascular.

Segundo a OMS, o AVC é a terceira maior causa de morte natural na população adulta no mundo, atrás do câncer e do infarto. No Brasil, o AVC representa a primeira causa de morte e incapacidade no País, o que cria grande impacto econômico e social, embora exista um declínio nas taxas de mortalidade. Dados de estudo nacional indicaram incidência anual de 108 casos por 100 mil habitantes, taxa de mortalidade de $18,5 \%$ aos 30 dias e de $30,9 \%$, aos 12 meses, com índice de recorrência após um ano de 15,9\% (BRASIL, 2013).

Atualmente, observa-se que o número de adultos jovens acometidos está aumentando consideravelmente, em função da exposição crescente aos fatores de riscos, como uso de contraceptivo oral, sedentarismo, obesidade, tabagismo e uso excessivo de bebidas alcoólicas (COSTA et al., 2008).

Estudos indicam aumento expressivo da morbidade de pessoas com alteraçốes físicas, auditivas, visuais e intelectuais após AVC (BRASIL, 2013). A incapacidade parcial ou completa ocorre entre $24 \%$ e $54 \%$ das ocorrências (OLIVEIRA; ORSINI, 2009). Conforme Barros (2003), 15\% não apresentam prejuízo de capacidade funcional, $37 \%$ mostram discreta alteraçáo e são capazes de realizar atividades de autocuidado, $16 \%$ apresentam moderada incapacidade, sendo semidependentes nas AVDs, e $32 \%$ demonstram sequelas graves, diminuindo consideravelmente sua capacidade funcional.

A alta prevalência de déficits neurológicos resultantes do AVC, decorrentes de comprometimentos cognitivos e/ou sensoriomotores, afetam os hábitos cotidianos temporariamente ou definitivamente, e implicam em mudança no estilo de vida, com repercussáo no próprio indivíduo e na família, dificultando ou impossibilitando o retorno ao trabalho, alterando a autonomia, a independência e a participação social (TOLDRÁ; SOUTO, 2013; STOFFEL; NICKEL, 2013; FERRO; LINS; TRINDADE FILHO, 2013; SANTOS, 2012).

Dessa forma, ressalta-se a importância deste tema, por ser uma das principais causas de incapacidades na população brasileira, que somada a outras comorbidades, leva a limitaçóes funcionais importantes, que podem comprometer diretamente a qualidade de vida das pessoas (THINEN; MORAES, 2013; ANDRADE et al., 2009).

A Terapia Ocupacional tem por objetivo desenvolver junto às pessoas com AVC atividades significativas e importantes, com a finalidade de propiciar maior independência, autonomia e condiçôes de participação social. Para tanto, é necessário que as pessoas com AVC recebam cuidados especializados de diferentes profissionais da área da saúde, com o enfoque multidisciplinar e integral, para garantir e atender, da melhor forma, as suas necessidades. Nesse sentido, os cuidados da Terapia Ocupacional abrangem as áreas ocupacionais que envolvem as atividades básicas e instrumentais da vida diária, bem como o trabalho, a educação, o brincar, o lazer e a participação social. Essas áreas podem variar de acordo com a idade da pessoa, o tipo de atividades, a rotina, os hábitos, dentre outros fatores. Diante disso, o principal foco do terapeuta ocupacional é o desempenho do ser humano nas diferentes açóes do cotidiano (CRUZ; TOYODA, 2009).

Em relação às práticas e aos modelos teóricos da Terapia Ocupacional, o estudo realizado por Santos (2007) sobre a atuação de Terapia Ocupacional em saúde física e contextos hospitalares assinalou uma variedade de atuações dos terapeutas ocupacionais, vinculadas a diferentes contextos de práticas e variadas abordagens terapêuticas, conforme as demandas das populaçóes atendidas. A Terapia Ocupacional vem se aperfeiçoando e demonstrando competência clínica e tecnológica em sua atuação, maximizando o potencial funcional das pessoas acompanhadas por esse terapeuta (GOLLEGÃ; LUZO; DE CARLO, 2001).

O presente estudo visa a contribuir para a sistematização do conhecimento produzido pela Terapia Ocupacional envolvendo o tema AVC, nos últimos dez anos (2003-2013). 


\section{Metodologia}

Trata-se de uma revisão integrativa da literatura, método comumente utilizado na Prática Baseada em Evidência (PBE). O método de revisão integrativa da literatura consiste na realização de uma análise ampla da literatura, para contribuir, por meio de discussôes sobre métodos e resultados de pesquisas, e reflexôes, para a realização de futuros estudos. O propósito inicial é obter um profundo entendimento de um determinado fenômeno, baseando-se em estudos anteriores (MENDES; SILVEIRA; GALVĀO, 2008).

O método permite sintetizar múltiplos estudos, de forma sistemática e ordenada, possibilitando conclusôes gerais acerca da área estudada. Segundo o método, a análise das pesquisas serve como um suporte para a tomada de decisão e a melhoria da prática clínica, sinaliza lacunas do conhecimento a serem preenchidas com a realização de novos estudos, torna os resultados de pesquisa mais acessíveis e permite ao leitor acesso às diversas pesquisas em um único estudo, amenizando algumas barreiras para a utilização do conhecimento científico (MENDES; SILVEIRA; GALVÃO, 2008; BOTELHO; CUNHA; MACEDO, 2011; SOUZA; SILVA; CARVALHO, 2010).

Conforme o método, para a realização da Revisão Integrativa, seguiu-se as seguintes etapas: seleção da questão norteadora da pesquisa; definição do objetivo específico; coleta de dados dentro dos critérios de inclusão e exclusão previamente estabelecidos; categorização; avaliação dos estudos incluídos; análise dos resultados, e síntese do conhecimento (MENDES; SILVEIRA; GALVÃO, 2008; SOUZA; SILVA; CARVALHO, 2010).

Para o norteamento do estudo, formulou-se a seguinte questão: Qual a produção científica dos terapeutas ocupacionais brasileiros, envolvendo o tema AVC, nos últimos dez anos (2003-2013)?

Para a realização do estudo, foram utilizados os seguintes descritores: "terapia ocupacional" em conjunto com outros descritores: "acidente vascular cerebral", "acidente vascular encefálico", "reabilitação" e "reabilitação neurológica". Foram consultadas as bases de dados nacionais Scientific Eletronic Library Online (SciELO Brasil), Literatura Latino-Americana e do Caribe de Ciências da Saúde (Lilacs) e Biblioteca Virtual em Saúde (Bireme). Foram também consultados os periódicos específicos da área de Terapia Ocupacional: Cadernos da Terapia Ocupacional da Universidade Federal de Sáo Carlos e Revista de Terapia Ocupacional da Universidade de São Paulo, pelo mecanismo automático, sendo que, nessa última revista, foi complementado com varredura manual. Outras duas revistas em que a área de Terapia Ocupacional publica foram consultadas: Revista O Mundo da Saúde, utilizando o mecanismo de varredura manual, e a Revista ACTA Fisiátrica, por busca automática.

Foram determinados como critérios de inclusão artigos nacionais sobre Terapia Ocupacional relacionados ao AVC e publicados em periódicos indexados no período entre 2003 e 2013 . Foram excluídos os artigos referentes à produção internacional, artigos fora do período determinado para o estudo e os que náo se relacionavam ao tema $\mathrm{AVC}$ e a Terapia Ocupacional. Após a coleta de dados, foi realizada organização em banco de dados e análise dos resultados encontrados.

\section{Resultados}

$\mathrm{Na}$ busca realizada nas bases de dados SciELO Brasil e Bireme, não foi encontrado nenhum artigo. A busca na base Lilacs localizou 12 artigos. Após a aplicação dos critérios de inclusão e exclusão, identificou-se que cinco artigos náo se referiam a produção de Terapia Ocupacional, não estavam relacionados ao tema ou estavam fora do período determinado para o estudo, totalizando, assim, sete artigos selecionados. Quanto aos periódicos nacionais consultados, foram identificados 15 artigos, porém cinco artigos repetiam os encontrados nas bases de dados, selecionando-se, então dez artigos. Desta forma, 17 artigos compuseram o estudo de revisão integrativa.

A partir dos resultados encontrados, é possível verificar que a produção científica nacional de Terapia Ocupacional sobre o tema AVC, em periódicos indexados, tem se demonstrado instável em relação ao número de publicaçóes. A Figura 1 apresenta os períodos de crescimento, compreendida entre os anos de 2004, 2010, 2012 e 2013, sendo o ano de 2013 com o maior número de publicações (4) e ausência de publicação em 2003, 2007, 2008 e 2009.

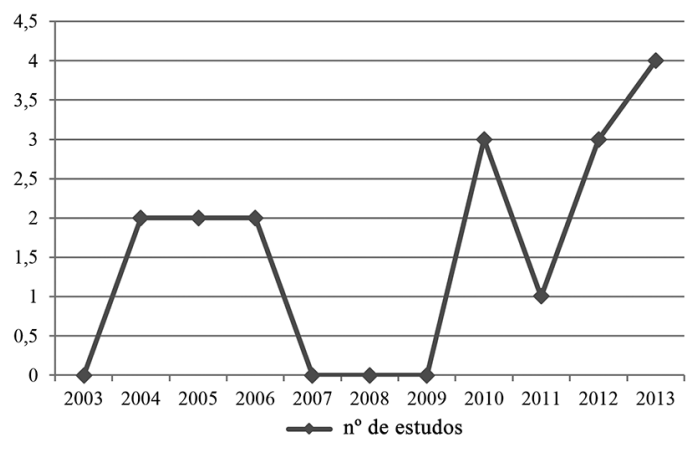

Figura 1. Distribuição de artigos por ano de publicação - 2003 a 2013. 
Quanto ao idioma, lembrando que todos os periódicos analisados são nacionais, 16 publicaçóes estão em português e apenas uma, em inglês.

Dentre as revistas científicas que publicaram sobre o assunto, destacam-se o Cadernos de Terapia Ocupacional da Universidade Federal de São Carlos (35\%) e a Revista Acta Fisiátrica (35\%), seguidos da Revista Mundo da Saúde (12\%), cuja somatória das publicaçóes correspondem a $82 \%$ do total das publicaçóes selecionadas. As demais revistas - Arquivos de Neuropsiquiatria, Revista de Fisioterapia e Pesquisa da USP e Revista de Terapia Ocupacional da USP - apresentam cada uma 6\%, conforme apresentado na Tabela 1.

A Tabela 2 mostra a distribuição dos artigos por título, autor(es), periódicos e ano de publicação. Com relação à autoria dos artigos selecionados, observou-se que quatro $(23,5 \%)$ estudos foram

Tabela 1. Distribuição de artigos científicos segundo o periódico das publicações e a base de indexação -2003 a 2013.

\begin{tabular}{lccc}
\hline \multicolumn{1}{c}{ PERIÓDICO } & BASE DE INDEXAÇÃO & $\mathbf{N}^{\mathbf{0}}$ & $\%$ \\
\hline Acta Fisiátrica & Lilacs & 6 & $35 \%$ \\
Arquivos de Neuropsiquiatria & Scielo & 1 & $6 \%$ \\
Cadernos de Terapia Ocupacional UFSCAR & Lilacs & 6 & $35 \%$ \\
Mundo da Saúde & Lilacs & 2 & $12 \%$ \\
Revista de Fisioterapia USP & Scielo & 1 & $6 \%$ \\
Revista de Terapia Ocupacional USP & Lilacs & 1 & $6 \%$ \\
TOTAL & - & 17 & 100 \\
\hline
\end{tabular}

Tabela 2. Artigos por título, autor(es), periódicos e ano de publicação.

\begin{tabular}{|c|c|c|c|c|}
\hline $\mathbf{N}^{\circ}$ & Título & Autor (es) & Periódicos & Ano \\
\hline 1 & $\begin{array}{l}\text { A terapia de restrição como forma de aprimoramento da } \\
\text { função do membro superior em pacientes com hemiplegia }\end{array}$ & Riberto et al. & Acta Fisiátrica & 2005 \\
\hline 2 & Acidente vascular cerebral crônico: reabilitação & Terranova et al. & Acta Fisiátrica & 2012 \\
\hline 3 & $\begin{array}{l}\text { Grupo de atividades de vida diária: influência do } \\
\text { procedimento em pacientes adultos com acidente vascular } \\
\text { encefálico isquêmico }\end{array}$ & Albuquerque et al. & Acta Fisiátrica & 2011 \\
\hline 4 & $\begin{array}{l}\text { A influência da negligência unilateral no desempenho de } \\
\text { atividades de vida cotidiana - relato de três casos }\end{array}$ & $\begin{array}{l}\text { Tsukimoto e } \\
\text { Valester }\end{array}$ & Acta Fisiátrica & 2005 \\
\hline 5 & $\begin{array}{l}\text { A interferência dos aspectos perceptocognitivos nas } \\
\text { atividades de vida diária e nas atividades instrumentais de } \\
\text { vida diária, em clientes com sequelas por lesão neurológica }\end{array}$ & Conti & Acta Fisiátrica & 2006 \\
\hline 6 & $\begin{array}{l}\text { Estimulação elétrica funcional otimizada em pacientes com } \\
\text { hemiparesia por doença cerebrovascular }\end{array}$ & Oshiro et al. & Acta Fisiátrica & 2012 \\
\hline 7 & $\begin{array}{l}\text { Evaluation of performance and personal satisfaction of the } \\
\text { patient with spastic hand after using a volar dorsal orthosis }\end{array}$ & $\begin{array}{l}\text { Garros, Gagliardi } \\
\quad \text { e Guzzo }\end{array}$ & $\begin{array}{c}\text { Arq. } \\
\text { Neuropsiq. }\end{array}$ & 2010 \\
\hline 8 & $\begin{array}{l}\text { A importância do cuidador em pacientes portadores de } \\
\text { acidente vascular cerebral }\end{array}$ & Une e Bianchin & $\begin{array}{l}\text { Cad. Ter. } \\
\text { Ocup. UFSCar }\end{array}$ & 2004 \\
\hline 9 & $\begin{array}{l}\text { Preditores de independência funcional nas atividades de } \\
\text { vida diária Pós-Acidente Vascular Encefálico }\end{array}$ & Cruz & $\begin{array}{l}\text { Cad. Ter. } \\
\text { Ocup. UFSCar }\end{array}$ & 2010 \\
\hline 10 & $\begin{array}{l}\text { Manual de orientação de posicionamento e execução de } \\
\text { atividade de vida diária para paciente com acidente vascular } \\
\text { cerebral }\end{array}$ & Thinen e Moraes & $\begin{array}{l}\text { Cad. Ter. } \\
\text { Ocup. UFSCar }\end{array}$ & 2013 \\
\hline 11 & $\begin{array}{l}\text { Atenção Ambulatorial de média complexidade em saúde e } \\
\text { reabilitação de pessoas com deficiência física no âmbito da } \\
\text { Terapia Ocupacional: Reflexões a partir da prática }\end{array}$ & Toldrá e Souto & $\begin{array}{l}\text { Cad. Ter. } \\
\text { Ocup. UFSCar }\end{array}$ & 2013 \\
\hline 12 & $\begin{array}{l}\text { Comprometimento cognitivo e funcional em pacientes } \\
\text { acometidos de acidente vascular encefálico: Importância } \\
\text { da avaliação cognitiva para intervenção na Terapia } \\
\text { Ocupacional }\end{array}$ & $\begin{array}{l}\text { Ferro, Lins e } \\
\text { Trindade Filho }\end{array}$ & $\begin{array}{l}\text { Cad. Ter. } \\
\text { Ocup. UFSCar }\end{array}$ & 2013 \\
\hline
\end{tabular}


Tabela 2. Continuação...

\begin{tabular}{|c|c|c|c|c|}
\hline $\mathbf{N}^{\circ}$ & Título & Autor (es) & Periódicos & Ano \\
\hline 13 & $\begin{array}{l}\text { A utilização da atividade como ferramenta no processo } \\
\text { de intervenção do terapeuta ocupacional em reabilitação } \\
\text { neurológica }\end{array}$ & Stoffel e Nickel & $\begin{array}{l}\text { Cad. Ter. } \\
\text { Ocup. UFSCar }\end{array}$ & 2013 \\
\hline 14 & $\begin{array}{l}\text { Avaliação da sensibilidade tátil superficial em adultos com } \\
\text { lesão do sistema central: implicações na assistência em } \\
\text { terapia ocupacional }\end{array}$ & Lima et al. & $\begin{array}{l}\text { O Mundo da } \\
\text { Saúde }\end{array}$ & 2006 \\
\hline 15 & $\begin{array}{l}\text { Relação entre os aspectos das alterações funcionais e seu } \\
\text { impacto na qualidade de vida das pessoas com sequelas de } \\
\text { Acidente Vascular Encefálico (AVE) }\end{array}$ & $\begin{array}{l}\text { Delboni, Malengo } \\
\text { e Schmidt }\end{array}$ & $\begin{array}{l}\text { O Mundo da } \\
\text { Saúde }\end{array}$ & 2010 \\
\hline 16 & $\begin{array}{l}\text { Os efeitos da toxina botulínica no tratamento da } \\
\text { espasticidade: uma revisão da literatura }\end{array}$ & Portella & $\begin{array}{l}\text { Rev. de Fisio. } \\
\text { USP }\end{array}$ & 2004 \\
\hline 17 & $\begin{array}{l}\text { Perfil sociodemográfico e áreas de desempenho ocupacional } \\
\text { afetadas em pacientes pós-ave atendidos por um serviço de } \\
\text { terapia ocupacional }\end{array}$ & Meneses et al. & $\begin{array}{l}\text { Rev. Ter. } \\
\text { Ocup. USP }\end{array}$ & 2012 \\
\hline
\end{tabular}

Tabela 3. Síntese do conhecimento segundo as categorias temáticas.

\begin{tabular}{|c|c|c|c|}
\hline $\begin{array}{c}\text { CATEGORIZAÇÃO } \\
\text { TEMÁTICA }\end{array}$ & TIPO DE ESTUDOS & PRODUÇÕES & $\begin{array}{c}\text { CONTRIBUIÇÃO DO } \\
\text { CONHECIMENTO }\end{array}$ \\
\hline $\begin{array}{l}\text { Fundamentação } \\
\text { Teórica/ Revisão } \\
\text { Bibliográfica }\end{array}$ & Estudos Bibliográficos & $\begin{array}{l}\text { Stoffel e Nickel (2013); } \\
\text { Terranova et al. (2012); } \\
\text { Cruz (2010); Portella et al. } \\
\text { (2004); Une e Bianchin } \\
\text { (2004). }\end{array}$ & $\begin{array}{c}\text { Fundamentam e } \\
\text { caracterizam o campo } \\
\text { de atuação da terapia } \\
\text { ocupacional junto às } \\
\text { pessoas com AVC. }\end{array}$ \\
\hline $\begin{array}{l}\text { Estratégias e } \\
\text { Intervenções } \\
\text { Terapêuticas }\end{array}$ & $\begin{array}{l}\text { Estudos Descritivos/ } \\
\text { Casos Clínicos }\end{array}$ & $\begin{array}{l}\text { Ferro, Lins e Trindade Filho } \\
\text { (2012); Oshiro et al. (2012); } \\
\text { Albuquerque et al. (2011); } \\
\text { Delboni, Malengo e Schmidt } \\
\text { (2010); Garros, Gagliardi e } \\
\text { Guzzo (2010); Riberto et al. } \\
\text { (2005); Lima et al. (2006). }\end{array}$ & $\begin{array}{c}\text { Abordam o uso de } \\
\text { diferentes tipos de } \\
\text { avaliação e de instrumentos } \\
\text { terapêuticos para o } \\
\text { tratamento de reabilitação } \\
\text { de pessoas com AVC. }\end{array}$ \\
\hline $\begin{array}{l}\text { Reflexão da Prática e } \\
\text { Análise de Relatos }\end{array}$ & $\begin{array}{c}\text { Estudos Exploratórios/ } \\
\text { Relatos de Experiência } \\
\text { e de Casos }\end{array}$ & $\begin{array}{l}\text { Thinen e Moraes (2013); } \\
\text { Toldrá e Souto (2013); } \\
\text { Meneses et al. (2012); } \\
\text { Conti (2006); Tsukimoto e } \\
\text { Valester (2005). }\end{array}$ & $\begin{array}{l}\text { Analisam experiências que } \\
\text { contribuem para a reflexão } \\
\text { sobre a prática profissional. }\end{array}$ \\
\hline
\end{tabular}

publicados por profissionais terapeutas ocupacionais; outros quatro $(23,5 \%)$, por profissionais de Terapia Ocupacional com profissionais de outras áreas (fisioterapeuta, médico, médico fisiatra e psicólogo); cinco (29\%) artigos foram publicados por profissionais e docentes terapeutas ocupacionais; três (18\%) artigos de autoria de docentes e acadêmicos de Terapia Ocupacional, e um (6\%), publicação de docente de Terapia Ocupacional.

A síntese do conhecimento produzido com a revisão integrativa da literatura encontra-se na Tabela 3. As temáticas abordadas nos artigos foram divididas nas seguintes categorias de análise: Fundamentação Teórica/Revisão Bibliográfica (cinco artigos - 29,5\%); Estratégias e Intervençôes
Terapêuticas (sete artigos - 41\%); Reflexão da Prática e Análise de Relato (cinco artigos - 29,5\%).

\section{Discussão}

A periodicidade da produção científica nacional de Terapia Ocupacional relacionada ao AVC, em periódicos nacionais indexados, tem se mostrado instável em relação ao número de publicaçóes. Destaca-se a ausência de publicação nos anos de 2003, 2007, 2008 e 2009, e em 2013, conta-se com o maior número de publicaçóes (quatro artigos). Quanto à autoria dos artigos, verifica-se que, dos 17 artigos, oito foram publicados pelos profissionais terapeutas ocupacionais, sendo que quatro de forma conjunta 
com profissionais de outras áreas. Os nove artigos restantes contam com autoria de docentes de Terapia Ocupacional, sendo que cinco de forma conjunta com profissionais da área, três com estudantes e uma publicação de docente como único autor. Os profissionais terapeutas ocupacionais participam ao todo de 13 artigos. É provável que o fato de a maioria dos autores ou coautores das publicaçóes serem profissionais terapeutas ocupacionais deve-se à temática envolver diretamente a prática clínica.

Quanto ao periódico de publicação, é visto que parte destas ocorreu em periódicos específicos de Terapia Ocupacional (41\%), considerados referências para o campo profissional no âmbito nacional; entretanto, a maioria (59\%) foi distribuída em outras quatro revistas, sendo duas destas com maior foco na reabilitação (Tabela 1). Isso pode ter ocorrido devido ao interesse dos terapeutas ocupacionais em dialogar sobre temas de interface com outros profissionais e, ainda, em divulgar a contribuição da Terapia Ocupacional no atendimento das necessidades específicas dessa população.

Os estudos publicados nos Cadernos de Terapia Ocupacional da UFSCar, Mundo da Saúde e Revista de Fisioterapia e Pesquisa da USP identificam-se como produçóes mais voltadas ao estudo qualitativo ou quantiqualitativo, apresentam conteúdos relevantes que apontam necessidades específicas da população e valorizam a contribuição da profissão. Os artigos científicos produzidos pelos terapeutas ocupacionais juntamente com outros profissionais, de modo geral, caracterizam-se por estudos quantitativos, uso de casos clínicos e aplicação de protocolos de tratamento, escalas e testes de avaliação, ou seja, temáticas de interface e maior interesse para as outras profissốes da saúde.

Todos os artigos deste estudo são da Região Sudeste, o que indica a concentraçáo da produçáo de conhecimento científico da Terapia Ocupacional, possivelmente pelo maior número de profissionais, de instituiçôes de ensino superior e de grandes hospitais; portanto, há maior oferta de cursos oferecimento de maior oferta de cursos de graduação, residências e aprimoramentos, conforme identificado em outro estudo (GALHEIGO; ANTUNES, 2008). Tal realidade favorece a realização de pesquisa, a produçấo de conhecimento e, por consequência, a publicação científica.

Para a análise dos resultados dessa revisão integrativa referente ao tema Terapia Ocupacional e AVC, foram selecionadas três categorias de análise. A primeira categoria Fundamentaçáo Teórica/Revisão Bibliográfica é composta por cinco estudos; a segunda categoria Estratégias e
Intervençóes Terapêuticas, com sete artigos, e a terceira categoria Reflexão da Prática e Análise dos Relatos, cinco artigos.

Na primeira categoria Fundamentaçáo Teórica/ Revisão Bibliográfica, um dos estudos objetivou a reflexão teórica e a discussão da prática do terapeuta ocupacional em reabilitação neurológica, com foco no uso de atividades como ferramenta de intervenção, por meio de revisão de literatura e análise de experiência na área (STOFFEL; NICKEL, 2013). Os outros quatro artigos buscaram realizar estudos bibliográficos que fornecem bases teóricas para apoiar a prática da Terapia Ocupacional junto aos pacientes pós-AVC, focalizando temas específicos, como fatores preditores de independência funcional nas atividades de vida diária (AVD) e atividades instrumentais de vida diária (AIVD) (CRUZ, 2010); a aplicação de diferentes técnicas terapêuticas no tratamento e na reabilitação de paciente com AVC crônico (TERRANOVA et al., 2012); os efeitos da toxina botulínica no tratamento da espasticidade (PORTELLA et al., 2004), e o papel do cuidador na reabilitação (UNE; BIANCHIN, 2004), os quais serão descritos detalhadamente a seguir.

No estudo de Stoffel e Nickel (2013), destaca-se a utilização das AVDs no processo de reabilitação neurológica como o principal recurso para minimizar as incapacidades e as deficiências, para estimular e desenvolver habilidades, níveis de atividades e participação do sujeito. Os autores buscaram subsídio na Classificação Internacional de Funcionalidade, Incapacidade e Saúde (CIF), em associação com o Modelo de Ocupação Humana e a Abordagem de Aprendizagem Motora para Recuperação do Comportamento Motor. O estudo aponta que o tratamento de reabilitação neurológica deve focar a autonomia no desempenho de AVD e na retomada da participaçáo, e ressalta a necessidade de maior fundamentação teórica na atuação do terapeuta ocupacional para garantir sua identidade e competência profissional (STOFFEL; NICKEL, 2013). A CIF é reconhecida pelos terapeutas ocupacionais como ferramenta útil, por favorecer o registro e a avaliaçáo dos resultados de forma padronizada, e o diálogo com demais profissionais (CONRAD et al., 2012; DARZINS; FONE; DARZINS, 2006). Aponta-se para a necessidade de associar a CIF com outras ferramentas avaliativas, que identifiquem o significado e as experiências subjetivas das atividades e fundamentem a prática clínica (CONRAD et al., 2012).

O segundo estudo buscou analisar os preditores de função para as AVDs pós-AVC. Foram apontados, como preditores: gênero, etnia, gravidade do AVC, espasticidade, tipos de tratamento precoce 
e componentes motores. Os instrumentos mais utilizados e considerados úteis para avaliação foram o Índice de Barthel, a Medida de Independência Funcional e o Instrumental Activities of Daily Living Questionnaire (IADLq). O autor recomenda pesquisas para verificar o efeito de tratamentos, estudos de casos clínicos randomizados e revisóes sistemáticas, bem como adaptaçôes transculturais de instrumentos de avaliação voltados às AVDs e AIVDs (CRUZ, 2010).

Conforme os estudos supracitados, destaca-se a importância das AVDs como um dos aspectos relevantes do processo terapêutico no contexto da Terapia Ocupacional. As atividades ocupacionais diárias se constituem como foco terapêutico, de ensino e de grande potencial para a pesquisa (HASSELKUS, 2006), na medida em que, através da ocupação, os terapeutas ocupacionais buscam aprimorar a saúde e o bem-estar das pessoas (LAW, 2002).

Nessa categoria, o terceiro estudo revisou artigos sobre o uso de técnicas terapêuticas no tratamento e na reabilitação de paciente com AVC crônico (mais de três meses). Para a recuperação da função do membro superior, destacam-se: a terapia de contenção induzida; a estimulação elétrica funcional; o biofeedback com eletromiografia; o treino bilateral, e a terapia assistida por robô. No que se refere ao uso de órteses, apesar da sua ampla utilização, para membro superior, em casos de AVC, os autores apontam que não há evidências suficientes para afirmar ou contestar a eficácia do uso de órteses de membro superior em adultos após AVC, dada a heterogeneidade dos estudos revisados. Para a recuperação da função dos membros inferiores, destaca-se o treino de marcha, o treino de equilíbrio associado à fisioterapia convencional, a estimulação elétrica transcutânea, o uso de estimulação elétrica e o biofeedback. Já o uso da realidade virtual, o treino de força muscular associado ao treino aeróbio e a simulação de atividades diárias propiciam melhora tanto na função de membros superiores como inferiores (TERRANOVA et al., 2012).

$\mathrm{O}$ quarto artigo estuda o efeito da aplicação da toxina botulínica para a redução da espasticidade. Seu efeito aumenta a amplitude de movimento e melhora o desempenho funcional. Os exercícios de fortalecimento, alongamento e estimulação elétrica funcional (FES) podem aumentar os efeitos terapêuticos da toxina botulínica antes não alcançados em pacientes com espasticidade crônica. No entanto, é preciso haver aplicaçôes repetidas para a manutenção do efeito (PORTELLA et al., 2004). Embora não seja atribuição da Terapia Ocupacional a aplicação de FES, identifica-se que o estudo é de autoria conjunta de fisioterapeutas e terapeutas ocupacionais.

Nos últimos dois estudos, verifica-se uma diversidade de aplicação de técnicas terapêuticas, voltadas à reabilitação funcional para o AVC. Esses periódicos indicam a importância do conhecimento específico da área profissional e a necessária formação continuada dos profissionais, para os cuidados na aplicação das diversas e novas técnicas de reabilitação.

O último artigo que compóe essa categoria aborda a importância do cuidador para pessoas com AVC. O estudo bibliográfico constatou o predomínio de cuidadoras informais mulheres e que o ato de cuidar do outro leva muitas famílias a se desorganizarem, gerando um grande sofrimento ao cuidador. O cotidiano do cuidador é modificado na medida em que fica à disposição do outro, com menos tempo para o próprio autocuidado, ocasionando desgastes emocionais, físicos e psicossociais, e restriçóes para melhorar a qualidade de vida e de convivência social. Destaca a preocupação da equipe de saúde em elaborar proposta para melhorar a qualidade de vida do cuidador e a importância dessa temática, ainda pouco explorada (UNE; BIANCHIN, 2004).

A categoria Estratégias e Intervençóes Terapêuticas inclui estudos descritivos/casos clínicos, formada por sete artigos, que abordam o uso de diferentes estratégias de avaliação (3) e de tratamento (4). Os estudos sobre avaliação abordam a cognição (FERRO; LINS; TRINDADE FILHO, 2013); a sensibilidade tátil (LIMA et al., 2006), e a relação das alteraçóes funcionais com a avaliação da qualidade de vida (DELBONI; MALENGO; SCHMIDT, 2010). Os estudos sobre estratégias de tratamento analisam a aplicação de grupo de AVD (ALBUQUERQUE et al., 2011); terapia de restrição (RIBERTO et al., 2005); estimulação elétrica funcional (OSHIRO et al., 2012) e uso de órtese (GARROS; GAGLIARDI; GUZZO, 2010).

Nessa categoria, o primeiro estudo analisa os resultados da avaliação cognitiva com 44 pacientes com AVC, entre 30 e 80 anos, com os testes: Miniexame do Estado Mental (MEEM), do relógio, de trilhas A e B, e a Escala Funcional Brazilian Older American Assessment Questionnaire (BOMFAQ). Identificou que as alteraçóes cognitivas tanto em adultos como em idosos comprometem a realização das atividades cotidianas e ressalta a importância da avaliçáo para o desenvolvimento de estratégias de reabilitação que favoreçam as atividades, a autonomia e a qualidade de vida (FERRO; LINS; TRINDADE FILHO, 2013). O segundo estudo aborda a avaliação de sensibilidade tátil superficial com a utilização dos Monofilamentos de Semmes-Weinstein, em 27 adultos com lesão no 
sistema nervoso central, para mapear a extensão e o grau das alteraçôes, com vistas a uma intervenção terapêutica criteriosa. Sugere que o resultado do teste e o conhecimento que o terapeuta possui de seu paciente favorecem uma maior compreensão de suas dificuldades e necessidades nas atividades do cotidiano. Destaca a interferência dos déficits cognitivos, perceptual, motor e psicossocial na avaliação da sensibilidade (LIMA et al., 2006).

O terceiro artigo da categoria Estratégias e Intervenção Terapêutica buscou relacionar as alteraçôes funcionais e o impacto na qualidade de vida com pacientes com sequelas de AVC. Utilizou as escalas de Medida de Independência Funcional (MIF) e a Escala de Qualidade de Vida Específica para AVC, com 15 pacientes com hemiplegia, com idade média de 65 anos. Mostrou que há somente uma correlação entre os domínios trabalho e produtividade, e autocuidado, com a MIF. Os homens têm melhor mobilidade que as mulheres; os solteiros, divorciados e viúvos têm melhor desempenho nos papéis sociais do que os casados; e os canhotos têm maiores escores nos papéis sociais devido à bilateralidade dos hemisférios cerebrais. Tais informaçôes favorecem o aprimoramento das intervençóes em Terapia Ocupacional (DELBONI; MALENGO; SCHMIDT, 2010).

$\mathrm{O}$ quarto estudo dessa categoria focaliza os benefícios funcionais alcançados por meio de grupo de AVD (dez participantes), avaliados por meio do Health Assessment Questionnarie (HAQ) e do Funcional Activities Questionnarie (FAQ). Os resultados mostraram eficácia no uso de grupo de AVD para a obtenção de ganhos funcionais, mesmo em pacientes crônicos, devido à motivação despertada pela troca e pelo compartilhamento de experiências. Ressalta que o uso de grupo reduz custos, com bons resultados (ALBUQUERQUE et al., 2011). O quinto estudo mostra o efeito do uso da terapia de restrição em pacientes (9) com hemiparesia, subagudos e crônicos, no membro superior afetado. $\mathrm{Na}$ avaliaçáo pela Medida de Independência Funcional (MIF), pelo Teste Motor de Wolf (TMW), pela Escala de Avaliação das Deficiências do AVC (EADAVC) e pela dinamometria de preensão, obtiveram-se ganhos na destreza manual e na independência funcional, após o uso de terapia de restrição (RIBERTO et al., 2005). O sexto artigo estuda o efeito da estimulação elétrica funcional (FES) em pacientes (3) com hemiparesia com esboço de movimento de dedos, com aplicação de duas semanas. O estudo mostrou aumento da força muscular isométrica de extensores de punho e redução de tônus com melhora na destreza manual, na amplitude de movimento e na função (OSHIRO et al., 2012). A recomendação realizada na categoria anterior também se verifica nesse estudo, em que se identifica que os autores são profissionais de diferentes categorias.

O sétimo e último estudo da categoria Estratégias e Intervençóes Terapêuticas avalia o efeito da órtese dorsal volar de punho e dedos, com 30 pacientes com mão espástica, com uso de 8 horas diárias. Para a análise do desempenho e da satisfação pessoal, foram utilizados a Medida Canadense de Desempenho Ocupacional e o teste de caixa e blocos. Verificou-se melhora no posicionamento, na prevençáo de deformidades e maior conforto ao paciente, demonstrando que o uso de órtese dorsal volar de punho e dedos após AVC é eficiente para o desempenho funcional e a satisfação do paciente (GARROS; GAGLIARDI; GUZZO, 2010).

Todos os artigos que compóem a segunda categoria demonstram o uso de uma ampla gama de instrumentos de avaliação e recursos, que podem ser utilizados pelo terapeuta ocupacional com pessoas com AVC, embora alguns não sejam específicos da área da Terapia Ocupacional. Destacam-se nessa categoria três estudos (FERRO; LINS; TRINDADE FILHO, 2013; LIMA et al., 2006; DELBONI; MALENGO; SCHMIDT, 2010), que apontam para a importância dos instrumentos de avaliaçáo na identificação das alterações, na compreensão das necessidades, na identificação do impacto nas atividades e na qualidade de vida, além, ainda, do planejamento da intervençấo.

Os cuidados em saúde requerem que a avaliçáo possa ser capaz de fornecer uma visão abrangente das condiçôes e necessidades das pessoas, bem como do potencial de desempenho nas atividades do cotidiano, a partir da relação dinâmica dos diferentes fatores (funcionais, sociais, ambientais, emocionais), na perspectiva dos envolvidos (RODRIGUES; ALVES, 2011). Assim, o processo de avaliação não pode se limitar a um instrumento específico, visto que a avalição é considerada o fundamento para a intervenção (ROCHA; BRUNELLO, 2011). Isso convoca os terapeutas ocupacionais a vislumbrarem possibilidades de uso combinado de estratégias de avaliação quantitativas e qualitativas.

Os demais quatro estudos (ALBUQUERQUE et al., 2011; RIBERTO et al., 2005; OSHIRO et al., 2012; GARROS; GAGLIARDI; GUZZO, 2010) buscam, por meio de instrumentos de avaliação, demonstrar a eficácia do tratamento. Instrumentos adequados permitem a identificação de dificuldades, auxiliam no monitoramento do nível de funcionalidade e aferem o progresso resultante das intervenções (QUINN et al., 2011). O processo avaliativo vislumbra a produção de dados; para tanto, o uso de testes 
válidos e padronizados possibilitam a sistematização dos resultados (RODRIGUES; ALVES, 2011) bem como sua comparação com outros estudos.

A terceira categoria Reflexão da Prática e Análise dos Relatos compreende estudos exploratórios referentes a relatos de experiência e de caso, totalizando cinco artigos relativos aos seguintes temas: experiência de atendimento ambulatorial na atenção de média complexidade (TOLDRÁ; SOUTO, 2013); uso de manual de orientação durante a internação hospitalar (THINEN; MOARES, 2013); relação do perfil sociodemográfico com as áreas de desempenho ocupacional e a prática da Terapia Ocupacional (MENESES et al., 2012); efeitos da negligência unilateral no desempenho das atividades (CONTI, 2006), e a influência das alteraçóes perceptocognitivas nas AVDs e AIVDs (TSUKIMOTO; VALESTER, 2005).

O primeiro artigo caracteriza a atenção ambulatorial no âmbito da média complexidade em saúde e da reabilitação de pessoas com deficiência, incluindo aquelas com AVC. Os autores destacam o uso de tecnologias leves e leves-duras, tais como atendimento grupal aliado à abordagem corporal, por meio do método Self-Healing e uso de recursos de baixo custo como estratégias para a reabilitação. Mostrou que o uso da estratégia grupal em conjunto com a corporal propiciou a melhora das capacidades físicas e da consciência corporal, favorecida pela maior autoconfiança adquirida durante o aprendizado e a troca grupal, com repercussão na convivência familiar e na participação social (TOLDRÁ; SOUTO, 2013).

Dois estudos dessa revisão ressaltam o uso de grupo como estratégia terapêutica e seus benefícios para a recuperação da função e da consciência corporal, e para o compartilhamento de experiências (TOLDRÁ; SOUTO, 2013; ALBUQUERQUE et al., 2011). O grupo é reconhecido como um dispositivo potencializador de encontros, de contato com o outro, de trocas e de confrontos (SAMEA, 2008), o que favorece o processo de reabilitação em sentido mais amplo.

O segundo estudo descreve o processo de orientação e de uso de manual com instruçóes sobre posicionamento e AVD, durante a internação hospitalar, com os familiares e pacientes com AVC. Os familiares reconheceram a utilidade do manual e as orientações modificaram a forma de cuidado. O manual contribui para o esclarecimento de dúvidas, a aplicação das orientaçôes em casa e evitar a interrupção no processo de reabilitação, após a alta hospitalar (THINEN; MOARES, 2013). A indicação de materiais educativos contribui para a comunicação em saúde e a adoçáo de medidas de prevençáo e de cuidado, o que favorece a autonomia e a corresponsabilidade pela própria saúde (BRASIL, 2010). Ademais, seu uso contribui para a reabilitaçáo precoce, tendo em vista que a intervenção no hospital, a partir das AVDs, implica no uso integrado de várias funçóes (motricidade, consciência, planejamento, sensibilidade, memória, volição e motivação) (ALBUQUERQUE; SEABRA; OTSU, 2012).

O terceiro estudo da categoria identifica o perfil sociodemográfico de pacientes pós-AVC, atendidos pelos terapeutas ocupacionais, e sua relação com as áreas de desempenho ocupacional. Foram identificadas 40 áreas-problemas no desempenho ocupacional, sendo as mais citadas: alimentação, marcha (autocuidado), cozinhar (produtividade) e escrever (atividades de lazer). Ressalta a importância do suporte familiar e social, e da adequada estrutura dos serviços de reabilitação, para que a pessoa possa alcançar o máximo potencial de reabilitação. Destaca que o enfoque de reabilitaçáo deve se dar nas áreas de desempenho afetadas e que o planejamento das estratégias de reabilitação nos serviços deve ser pautado no conhecimento do perfil da populaçáa (MENESES et al., 2012).

O quarto estudo, pertencente à categoria Reflexão da Prática e Análise dos Relatos, discute que a negligência unilateral (NU), quando associada às alteraçôes motoras, cognitivas e emocionais, dificulta a realização das atividades cotidianas e a recuperação funcional, e aumenta o grau de dependência dos pacientes com AVC. Devido à falta de compreensão precisa sobre o efeito da NU durante as atividades, os autores enfatizam a necessidade de um protocolo de avaliação para a análise do desempenho das atividades e da programação de reabilitação para uma melhor recuperação (TSUKIMOTO; VALESTER, 2005). Tal estudo reafirma aspectos sobre a importância da avaliação no processo terapêutico, já apontados nos artigos pertencentes à segunda categoria.

O quinto e último estudo da categoria Reflexão da Prática e Análise dos Relatos mostra a interferência dos aspectos perceptocognitivos no desempenho das AVDs e AIVDs devido à lesão neurológica. Destaca que é essencial o conhecimento de tais alteraçôes, pois estas determinam o potencial de aprendizagem, a evolução do tratamento e a independência do cliente e da família (CONTI, 2006). Embora o artigo tenha sido apresentado como estudo bibliográfico, observou-se que se trata mais propriamente de relatos de casos para a abordagem do tema e não propriamente um estudo bibliográfico.

A presente revisão integrativa contribui, como um primeiro passo, para a construçáo do conhecimento 
científico sobre a temática estudada. Pode-se dizer que os estudos que formaram as três categorias temáticas enfatizaram as contribuiçóes da Terapia Ocupacional no tratamento de pessoas com AVC e apontaram para diferentes aspectos que favorecem a melhora da funcionalidade e a promoção da qualidade de vida. Apesar de alguns estudos mencionarem açóes ou resultados junto aos familiares/cuidadores, poucos focalizaram mais especificamente a rede social mais próxima, dado o seu papel na recuperação e na convivência/participação social das pessoas com AVC. Somente um estudo abordou a importância do cuidado com o cuidador.

Reconhece-se a importância dos relatos de experiências para a construçáo e a troca de conhecimento, para a análise mais aprofundada do impacto da deficiência/ incapacidade e seu significado na vida das pessoas. Vale destacar a contribuição desses estudos para o entendimento das técnicas terapêuticas, que podem, sobremaneira, qualificar o tratamento, ampliar a compreensáo das necessidades e possibilitar a reflexão sobre as especificidades da Terapia Ocupacional.

Muitos estudos assinalam a necessidade de uma maior sistematização do conhecimento para demonstrar a eficácia das açôes da Terapia Ocupacional junto às pessoas com AVC. Os autores Mendes, Silveira e Galvão (2008) ressaltam que um dos objetivos da PBE é encorajar a utilização dos resultados de pesquisa junto à assistência à saúde, para reafirmar a importância da pesquisa para a clínica. Verifica-se uma tendência de valorização de estudos sistematizados sobre as intervençôes, para demonstrar a eficácia do tratamento, mensurar as intervençóes e dar suporte para outros estudos na área de Terapia Ocupacional (SANTOS; DE CARLO, 2013).

Por fim, uma dificuldade que pode ser assinalada neste estudo é a inexistência de um descritor comum entre as publicações, o que torna o processo de pesquisa mais difícil, tendo em vista que as publicações são da área de Terapia Ocupacional, mas nem todas utilizam como descritor a Terapia Ocupacional. Isso aponta para a necessidade de que os terapeutas ocupacionais reconheçam a importância da utilizaçáo da denominação da profissão, para que os estudos possam ser identificados não só pela área, mas também por outras profissōes.

\section{Considerações finais}

A presente revisão integrativa da literatura apresentou uma síntese do conhecimento científico de Terapia Ocupacional produzido no Brasil, relacionado ao AVC, o que possibilitou um panorama e um conhecimento mais amplo sobre o tema e as tendências das produçóes no período de 2003-2013.
Diferentes modalidades de estudos devem ser estimuladas para abordar a temática proposta. A produção científica recente em Terapia Ocupacional voltada para a reflexão da prática contribui para a maior divulgação e melhor desenvolvimento das práticas terapêuticas voltadas ao cuidado das pessoas com AVC e um maior reconhecimento do papel do terapeuta ocupacional. No entanto, recomenda-se o emprego de métodos qualitativos mais específicos, que permitam uma análise mais aprofundada na forma de estudos de casos, de serviços e outras modalidades, que favoreçam a compreensão das necessidades da população e a reflexão das especificidades e da contribuiçáo da profissáo. Também se observou um maior número de estudos que apontam para a importância do uso de instrumentos de avaliação e técnicas de tratamento, que correspondem às necessidades $\mathrm{da}$ prática profissional no campo da reabilitação, os quais necessitam de aprimoramento, para atender os padrôes científicos internacionalmente reconhecidos, conforme a tendência das práticas de saúde.

Considerando-se que o estudo sobre Terapia Ocupacional com pessoas com AVC é um tema amplo, é importante a utilização de descritor que especifique a profissão para facilitar a localização da produção científica. Como limites do estudo, são apontados o número de descritores utilizado e o fato de o estudo abranger exclusivamente a produção científica nacional. Sugere-se que outras pesquisas possam ser feitas a partir do uso de novos descritores e incluam a literatura internacional, para o estudo da temática.

O estudo demonstra, ainda, a necessidade de intensificar a realização de pesquisas para divulgação do conhecimento produzido pela Terapia Ocupacional e seu reconhecimento no campo da reabilitaçáo de pessoas com AVC, tendo em vista a importante atuação da Terapia Ocupacional com essa população.

\section{Referências}

ALBUQUERQUE, C. P. et al. Grupo de atividades de vida diária: influencia do procedimento em pacientes adultos com acidente vascular encefálico isquêmico. Acta Fisiátrica, São Paulo, v. 18, n. 2, p. 71-74, 2011.

ALBUQUERQUE, S. H.; SEABRA, A. D.; OTSU, A. E. Atividades de vida diária com pacientes internados. In: CRUZ, D. M. C. Terapia Ocupacional na reabilitação pós-acidente vascular encefálico. São Paulo: Santos, 2012. p. 229-243.

ANDRADE, L. M. et al. A problemática do cuidador familiar do portador de acidente vascular cerebral. Revista da Escola de Enfermagem da USP, São Paulo, v. 43, n. 1, p. 37-43, 2009. 
BARROS, J. E. F. Doença encefalovascular. In: NITRINI, R.; BACHESCHI, L. A. A neurologia que todo médico deve saber. São Paulo: Atheneu, 2003. p. 171-188.

BRASIL. Ministério da Saúde. Secretaria de Vigilância em Saúde. Secretaria de Atenção à Saúde. Política $N a$ cional de Promoção da Saúde. Brasília, 2010. Disponível em: <http://bvsms.saude.gov.br/bvs/publicacoes/politica_nacional_promocao_saude_3ed.pdf>. Acesso em: 22 nov. 2010.

BRASIL. Ministério da Saúde. Secretaria de Atenção à Saúde. Departamento de Açôes Programáticas Estratégicas. Diretrizes de atenção à reabilitação da pessoa com acidente vascular cerebral. Brasília, 2013. Disponível em: <http://bvsms.saude.gov.br/bvs/publicacoes/diretrizes_ atencao_reabilitacao_acidente_vascular_cerebral.pdf $>$. Acesso em: 01 ago. 2015.

BOTELHO, L. L. R.; CUNHA, C. C. A.; MACEDO, $\mathrm{M}$. O método da revisão integrativa nos estudos organizacionais. Gestão e Sociedade, Belo Horizonte, v. 5, n. 11, p. 121-136, 2011.

CRUZ, D. M. C.; TOYODA, C. Y. Terapia Ocupacional no tratamento do AVC. ComCiência, Campinas, n. 109, p. 1-5, 2009.

CRUZ, D. M. C. Preditores de independência funcional nas atividades de vida diária pós-Acidente Vascular Encefálico. Cadernos de Terapia Ocupacional da UFSCar, São Carlos, v. 18, n. 3, p. 275-286, 2010.

CONRAD, A. et al. Validation of the comprehensive ICF core set for multiple sclerosis from the perspective of occupational therapists. Scandinavian Journal of Occupational Therapy, Munich, v. 19, n. 6, p. 468-487, 2012.

CONTI, J. A interferência dos aspectos percepto-cognitivos nas atividades de vida diária e nas atividades instrumentais de vida diária em clientes com sequelas por lesão neurológica. Acta Fisiátrica, São Paulo, v. 13, n. 2, p. 83-86, 2006.

COSTA, F. et al. Nível de conhecimento da população adulta sobre acidente vascular cerebral (AVC) em Pelotas - RS. Jornal Brasileiro Neurocirurgia, Curitiba, v. 19, n. 1, p. 31-37, 2008.

DARZINS, P.; FONE, S.; DARZINS, S. The International classification of functioning, disability and health can help to structure and evaluate therapy. Australian Occupational Therapy Journal, Melbourne, v. 53, n. 2, p. 127-131, 2006. http://dx.doi.org/10.1111/j.14401630.2006.00580.x.

DELBONI, M. C. C.; MALENGO, P. C. M.; SCHIMIDT, E. P. R. Relação entre os aspectos das alterações funcionais e seu impacto na qualidade de vida das pessoas com sequelas de Acidente Vascular Encefálico (AVE). Mundo da Saúde, São Paulo, v. 42, n. 2, p. 165-175, 2010 .

FERRO, A. O.; LINS, A. E. S.; TRINDADE FILHO, E. M. Comprometimento cognitivo e funcional em pacientes acometidos de acidente vascular encefálico: importância da avaliação cognitiva para intervenção na
Terapia Ocupacional. Cadernos de Terapia Ocupacional da UFSCar, São Carlos, v. 21, n. 3, p. 521-527, 2013. http://dx.doi.org/10.4322/cto.2013.054.

GAGLIARDI, R. J. Acidente Vascular Cerebral ou Acidente Vascular Encefálico? Qual a melhor nomenclatura? Revista Neurociências, São Paulo, v. 18, n. 2, p. 13132, 2010.

GALHEIGO, S. M.; ANTUNES, J. R. A caracterização da produção bibliográfica nas práticas hospitalares em terapia ocupacional no Brasil: uma revisão da literatura de 1990 a 2007. Revista de Terapia Ocupacional da Universidade de São Paulo, São Paulo, v. 19, n. 2, p. 91-99, 2008.

GARROS, D. S. C.; GAGLIARDI, R. J.; GUZZO, R. A. R. Evalution of performance and personal satisfaction of the patient with spatic hand after using a volar dorsal orthosis. Arquivos de Neuro-Psiquiatria, São Paulo, v. 68, n. 3, p. 385-389, 2010.

GOLlEGÃ, A. C. C.; LUZO, M. C. M.; DE CARLO, M. M. R. P. Terapia Ocupacional - princípios, recursos e perspectivas em reabilitação física. In: DE CARLO, M. M. R. P.; BARTALOTTI, C. Terapia Ocupacional no Brasil: fundamentos e perspectivas. São Paulo: Plexus, 2001. p. 137-154.

HASSELKUS, B. R. The world of everyday occupation: real people, real lives. The American Journal of Occupational Therapy, New York, v. 60, n. 6, p. 627640, 2006. http://dx.doi.org/10.5014/ajot.60.6.627. PMid:17153568.

INSTITUTO BRASILEIRO GEOGRÁFICA E ESTATÍSTICA - IBGE. Indicadores sociodemográfico e de saúde no Brasil. Rio de Janeiro, 2009. (Estudos \& Pesquisas: Informação Demográfica e Socioeconômica, 25). Disponível em: <http://www.ibge.gov.br/home/estatistica/populacao/indic_sociosaude/2009/indicsaude.pdf $>$. Acesso em: 01 nov. 2014

LAW, M. Participation in the occupations of everyday life. The American Journal of Occupational Therapy, New York, v. 56, n. 6, p. 640-649, 2002. http://dx.doi. org/10.5014/ajot.56.6.640. PMid:12458856.

LIMA, S. M. P. F. et al. Avaliação da sensibilidade tátil superficial em adultos com lesão do sistema central: implicações na assistência em terapia ocupacional. Mundo da Saúde, São Paulo, v. 30, n. 1, p. 73-80, 2006.

MENDES, K. D.; SILVEIRA, R. C. C. P.; GALVÃO, C. M. Revisão Integrativa: método de pesquisa para a incorporação de evidências na saúde e na enfermagem. Texto Contexto Enfermagem, Florianópolis, v. 14, n. 4 , p. 758-764, 2008.

MENESES, K. V. P. et al. Perfil sócio demográfico e áreas de desempenho ocupacional afetadas em pacientes pós-AVE atendidos por um serviço de terapia ocupacional. Revista de Terapia Ocupacional da Universidade de São Paulo, São Paulo, v. 23, n. 2, p. 107-112, 2012.

OLIVEIRA, M. R.; ORSINI, M. Escalas de avaliação da qualidade de vida em pacientes brasileiros após aci- 
dente vascular cerebral. Revista Neurociência, São Paulo, v. 17, n. 3, p. 255-262, 2009.

ORGANIZAÇÃO MUNDIAL DA SAÚDE - OMS. O Manual STEPS de Acidentes Vascular Cerebrais da $O M S$ : enfoque passo a passo para a vigilância de acidentes vascular cerebrais. Genebra, 2005. Disponível em: <http://new.paho.org/hq/dmdocuments/2009/manualpo.pdf>. Acesso em: 29 nov. 2014.

OSHIRO, S. H. et al. Estimulação elétrica funcional otimizada em pacientes com hemiparesia por doença cerebrovascular. Acta Fisiátrica, Sáo Paulo, v. 19, n. 1, p. 46-49, 2012.

PORTELLA, L. V. et al. Os efeitos da toxina botulínica no tratamento da espasticidade: um revisão de literatura. Revista de Fisioterapia da Universidade de São Paulo, São Paulo, v. 11, n. 1, p. 47-55, 2004.

QUINN, T. J. et al. Functional assessment in older people. BMJ, Glasgow, v. 343, p. 469-473, 2011. PMid:21859792.

RIBERTO, M. et al. A terapia de restrição como forma de aprimoramento da função do membro superior em pacientes com hemiplegia. Acta Fisiátrica, São Paulo, v. 12, n. 1, p. 15-19, 2005.

ROCHA, E. F.; BRUNELLO, M. I. B. Avaliação qualititativa em Terapia Ocupacional: princípios, métodos e técnicas de coleta de dados. In: SOUZA, A. C. A.; GALVÂO, C. R. C. Terapia Ocupacional: fundamentação e prática. Rio de Janeiro: Guanabara Koogan, 2011. p. 44-48.

RODRIGUES, A. M. V. N.; ALVES, G. B. O. Avaliação dos Componentes de desempenho sensorial e neuromuscular. In: SOUZA, A. C. A.; GALVÃO, C. R. C. Terapia Ocupacional: fundamentaçáo e prática. Rio de Janeiro: Guanabara Koogan, 2011. p. 74-94.

SAMEA, M. O dispositivo grupal como intervenção em reabilitação: reflexôes a partir da prática em Terapia Ocupacional. Revista de Terapia Ocupacional da Universidade de São Paulo, São Paulo, v. 19, n. 2, p. 85-90, 2008.

SANTOS, C. A. V. O serviço de terapia ocupacional em contextos hospitalares, sob a perspectiva dos usuários. 2007. 76 f. Monografia (Graduaçáo em Terapia Ocupacional) - Universidade de São Paulo, Ribeirão Preto, 2007.
SANTOS, C. A. V.; DE CARLO, M. M. R. P. Hospital como campo de práticas: revisáo integrativa da literatura e a terapia ocupacional. Cadernos de Terapia Ocupacional da UFSCar, São Carlos, v. 21, n. 1, p. 99-107, 2013. http://dx.doi.org/10.4322/cto.2013.014.

SANTOS, E. B. Estresse percebido dos idosos após o Acidente Vascular Cerebral. 2012. 124 f. Dissertação (Mestrado em Ciências) - Universidade de São Paulo, Ribeirão Preto, 2012.

SOUZA, M. T.; SILVA, M. D.; CARVALHO, R. Revisão integrativa: o que é e como fazer. Einstein, São Paulo, v. 8, n. 1, p. 102-106, 2010. http://dx.doi.org/10.1590/ S1679-45082010RW1134.

STOFFEL, D. P.; NICKEL, R. A utilização da atividade como ferramenta no processo de reabilitação de intervenção do terapeuta ocupacional em reabilitaçáo neurológica. Cadernos de Terapia Ocupacional da UFSCar, São Carlos, v. 21, n. 3, p. 617-622, 2013. http://dx.doi. org/10.4322/cto.2013.064.

TERRANOVA, T. T. et al. Acidente Vascular Cerebral Crônico: reabilitação. Acta Fisiátrica, São Paulo, v. 19, n. 2, p. 50-59, 2012.

THINEN, N. C.; MORAES, A. C. F. Manual de orientação de posicionamento e execuçáo de atividades de vida diária para pacientes com acidente vascular cerebral. Cadernos de Terapia Ocupacional da UFSCar, São Carlos, v. 21, n. 1, p. 131-139, 2013. http://dx.doi. org/10.4322/cto.2013.017.

TOLDRÁ, R. C.; SOUTO, A. C. F. Atenção Ambulatorial de média complexidade em saúde e reabilitação de pessoas com deficiência física no âmbito da Terapia Ocupacional: Reflexóes a partir da prática. Cadernos de Terapia Ocupacional da UFSCar, São Carlos, v. 21, n. 2, p. 299-306, 2013. http://dx.doi.org/10.4322/ cto.2013.031.

TSUKIMOTO, D. R.; VALESTER, G. A. A influência da negligencia unilateral no desempenho de atividade de vida cotidiana - relato de 3 casos. Acta Fisiátrica, São Paulo, v. 12, n. 3, p. 108-114, 2005.

UNE, D. S.; BIANCHIN, M. A. A importância do cuidador em pacientes portadores de Acidente Vascular Cerebral. Cadernos de Terapia Ocupacional da UFSCar, São Carlos, v. 12, n. 2, p. 101-104, 2004.

\section{Contribuição dos Autores}

Concepção do texto: Carolina Gina Shin e Rosé Colom Toldrá. A primeira autora realizou a organização de fontes, análises e redação do texto, e a segunda autora participou das análises e realizou a revisão final do texto. Todas as autoras aprovaram a versão final do artigo.

\section{Notas}

${ }^{1}$ O estudo é produto do Trabalho de Conclusão do Curso de Terapia Ocupacional da Faculdade de Medicina da Universidade de São Paulo em 2014.

${ }^{2}$ Embora o termo Acidente Vascular Encefálico (AVE) venha sendo bastante utilizado atualmente, por reconhecer outras estruturas encefálicas que podem ser acometidas pela lesão, foi adotado, neste artigo, o termo Acidente Vascular Cerebra (AVC), com base na recomendação da Sociedade Brasileira de Doenças Cerebrovasculares, por ser considerado o termo mais conhecido, divulgado e mais empregado em comparação à denominação AVE (GAGLIARDI, 2010). 